PESSOA, AMS; RÊGO, ER; RÊGO, MM. 2021. Additive and non-additive genetic effects for fruit traits of ornamental pepper. Horticultura Brasileira 39: 039-045. DOI: http://dx.doi.org/10.1590/s0102-0536-20210106

\title{
Additive and non-additive genetic effects for fruit traits of ornamental pepper
}

\author{
Angela Maria dos S Pessoa ${ }^{1} \mathbb{D}$; Elizanilda R do Rêgo ${ }^{1 \mathbb{D}}$; Maílson $M$ do Rêgo ${ }^{1 \mathbb{D}}$ \\ ${ }^{1}$ Universidade Federal da Paraíba, Centro de Ciências Agrárias (UFPB-CCA), Areia-PB, Brasil, elizanilda@cca.ufpb.br; pbalegna@gmail.com
}

\begin{abstract}
Pepper plants can be used as ornamental ones due to the contrasting traits of plants, flowers, and especially fruits. This variability can be exploited in genetic improvement programs aiming at identifying superior genotypes and obtaining hybrids. This work aimed to select parental and hybrid individuals through diallel analysis for fruit traits in Capsicum annuum. Seven pepper genotypes were crossed, and eleven fruit-related quantitative traits were evaluated. The data were subjected to diallel analysis using Griffing's Method 1 (fixed model). There was a predominance of additive genetic effects on the fruit traits of ornamental pepper plants. Genotypes UFPB77.3 and UFPB390 are indicated as parents for the improvement of ornamental pepper plants, for fruit traits. Hybrids UFPB77.3 x UFPB099, UFPB390 x UFPB77.3, UFPB390 x UFPB001, UFPB390 x UFPB099, UFPB134 $x$ UFPB77.3, and UFPB134 x UFPB390 present a potential for the production of hybrids and generation of segregating populations of ornamental peppers.
\end{abstract}

Keywords: Capsicum annuum, diallel, hybridization.

\section{RESUMO}

Efeitos genéticos aditivos e não aditivos para características de frutos de pimenteira ornamental

As pimenteiras podem ser usadas como plantas ornamentais devido às características contrastantes de plantas, flores e especialmente frutos. Esta variabilidade pode ser explorada em programas de melhoramento genético, com o objetivo de identificar genótipos superiores e obter híbridos. Este trabalho teve como objetivo selecionar indivíduos parentais e híbridos por meio da análise dialélica de caracteres de frutos em Capsicum annuum. Sete genótipos de pimenteiras foram cruzados e onze características quantitativas relacionadas aos frutos foram avaliadas. Os dados foram submetidos à análise dialélica pelo método 1 de Griffing (modelo fixo). Houve predomínio de efeitos genéticos aditivos nas características de frutos em plantas de pimenteiras ornamentais. Os genótipos UFPB77.3 e UFPB390 são indicados como pais para o melhoramento de pimenteiras ornamentais, para características de frutos. Os híbridos UFPB77.3 x UFPB099, UFPB390 x UFPB77.3, UFPB390 x UFPB001, UFPB390 x UFPB099, UFPB134 x UFPB77.3 e UFPB134 x UFPB390 apresentam potencial para produção de híbridos e geração de populações segregantes de pimenteiras ornamentais.

Palavras-chave: Capsicum annuum, dialelo, hibridação.

\section{Received on July 1, 2020; accepted on February 9, 2021}

$\mathrm{P}$ eppers (Capsicum spp.) belong to the genus Capsicum, family Solanaceae (Bosland \& Votava, 2012), and include nearly 30 known species (Pickersgill, 1997), of which only five are cultivated (Heiser \& Pickersgill, 1969).

C. annuum is the most commercialized species in the world (Rêgo \& Rêgo, 2016), presenting great use versatility, both in food (Yamamoto \& Nawata, 2005) and in medicine, with the identification of chemical compounds, such as capsaicin (Perucka \& Materska, 2001). Capsaicin presents several health benefits, such as pain relief, anti-inflammatory action, thermogenic effects (Srinivasana, 2015), and analgesic effect (Romero et al., 2019). C. annuum can also be used as an ornamental plant (Rêgo \& Rêgo, 2018; Nascimento et al., 2019a). Pepper production is important for agribusiness in the whole world, in which this market stimulates family agriculture and increases jobs and income generation (Camara, 2020).

The use of pepper plants for ornamental purposes occurs due to its contrasting phenotypic aspects (Pessoa et al., 2019a; Fortunato et al., 2019), flowers (Pessoa et al., 2019b), and mainly fruits. The fruits present different sizes, shapes, and maturation stages, with several color shades (Nascimento et al., 2019b). These differences make this species attractive as an ornamental fruit plant. This variability can be exploited in genetic improvement programs to identify superior genotypes, obtain hybrids, and develop segregating populations.

The analysis of the combining ability of potential parents allows identifying those more fit to transmit the desirable traits to the offspring, as well as aiding in the choice of more promising crossings for commercial use, through the release of hybrids (Souza et al., 2013). Thus, this work aimed to select parental and hybrid individuals through diallel analysis for fruit traits in ornamental peppers. 


\section{MATERIAL AND METHODS}

The research was performed in a plant nursery at the Center of Agricultural Sciences of the Federal University of Paraíba (UFPB), Paraíba state, Brazil.

Seven ornamental pepper cultivars (C. anпиит), (UFPB001, UFPB004, UFPB77.3, UFPB099, UFPB134, UFPB137 and UFPB390), were crossed in a complete diallel scheme. The manual crossings were performed in the pre-anthesis phase (Rêgo et al., 2012; Nascimento et al., 2012). The fruits were collected when ripe and the seeds were removed.

The seeds of the parents and 42 hybrids were sown in polystyrene trays with 128 cells filled with a commercial substrate $\left(\right.$ Plantmax $\left.^{\circledR}\right)$. Thirty days after germination, the seedlings were transferred to plastic pots containing $900 \mathrm{~mL}$ of the same substrate.

The morpho-agronomical characterization was performed based on the list of descriptors followed by IPGRI (1995). The following traits were evaluated: Days to fructification, Number of fruits per plant, Fruit weight, Fruit length, Largest fruit diameter, Smallest fruit diameter, Pedicel length, Pericarp thickness, Placenta length, Number of seeds per fruit, and Dry matter content. The tools used for data collection were a digital pachymeter (Leetools ${ }^{\circledR}$ digital pachymeter) and an analytical balance (Bel engineering $\left.{ }^{\circledR}\right)$.

The experimental design was completely randomized, with 49 treatments (parents and hybrids) and eight replications. The general combining ability (GCA) and the specific combining ability (SCA) were estimated using Griffing's method 1 (1956) (fixed model). All statistical and genetic analyses were performed using the Genes software (Cruz, 2006).

\section{RESULTS AND DISCUSSION}

The traits were significant by the $\mathrm{F}$ test $(p \leq 0.01)$ for treatments and general combining ability (data not shown). The selection of parents is the first step of the breeding process, and the diallelic analysis of these constitutes the most promising prediction method for the determination of superior parents (Pimentel et al., 2013). The use of genotypes that contrast with each other is essential for genetic improvement since genetic variability is widened, favoring selection (Ramalho et al., 2008). Such as in the present work, Rêgo et al. (2009), in a study with diallel crosses on the yield and fruit quality traits of $C$. baccatum, reported significant GCA effects for all fruit characters.

The specific combining ability was significant $(\mathrm{p} \leq 0.01)$ for all traits, except the dry matter content (data not shown). The evaluated hybrids presented distinct behaviors for the traits and similarity only for the dry matter content.

The reciprocal effect was significant $(p \leq 0.01)$ for the evaluated traits, except for the pericarp thickness and the dry matter content, demonstrating that the phenotypes are influenced by the female parent. Nascimento et al. (2014) found significant reciprocal effects for the fruit traits through diallel analysis in $C$. annuum. The reciprocal effect indicates which genotype can be used as a female parent and in a hybrid combination (Baldissera et al., 2012).

The quadratic components for the GCA presented superior values for the number of fruits per plant, fruit weight, fruit length, largest fruit diameter, smallest fruit diameter, pericarp thickness, placenta length, and the number of seeds per fruit, indicating the presence of genetic variance and relative importance of additive gene action in trait heritability (Neves et al., 2014). The parents that presented additive gene effects are the most favorable for use in genetic improvement programs whose purpose is the selection of new pure lineages in advanced generations (Pimentel et al., 2013; Valadares et al., 2019).

The traits days to fructification, pedicel length, and fruit dry matter content presented superior values of SCA effects $\left(\varphi_{s}^{2}\right)$, indicating the predominance of non-additive gene action in the control of these traits as

Table 1. Estimates of the effects of the general combining ability $\left(\hat{\mathrm{g}}_{\mathrm{f}}\right)$ referring to fruit traits in Capsicum annuum. Areia, UFPB, 2019.

\begin{tabular}{|c|c|c|c|c|c|c|}
\hline \multirow{2}{*}{$\begin{array}{l}\text { Genotypes } \\
\text { (UFPB) }\end{array}$} & \multicolumn{6}{|c|}{ Characters } \\
\hline & DFR & NFP & FW & FL & LFD & SFD \\
\hline 001 & $3.33 * *$ & $-19.39 * *$ & $1.99 * *$ & $0.45 * *$ & $0.42 * *$ & $0.19 * *$ \\
\hline 004 & $0.72 *$ & $-17.14 * *$ & $2.54 * *$ & $0.80 * *$ & $0.40 * *$ & $0.22 * *$ \\
\hline 77.3 & $-2.19 * *$ & $30.22 * *$ & $-2.41 * *$ & $-0.97 * *$ & $-0.45^{* *}$ & $-0.17 * *$ \\
\hline 099 & $0.97 * *$ & $-15.93 * *$ & $1.95 * *$ & $0.81 * *$ & $0.31 * *$ & $0.13^{* *}$ \\
\hline 134 & $1.95 * *$ & $3.05^{*}$ & $-1.05^{* *}$ & $-0.29 * *$ & $-0.15^{* *}$ & $-0.12 * *$ \\
\hline 137 & $-1.33^{* *}$ & $-2.42 *$ & $-0.84 * *$ & $-0.12 * *$ & $-0.10^{* *}$ & $-0.04 * *$ \\
\hline \multirow[t]{2}{*}{390} & $-3.44 * *$ & $21.59 * *$ & $-2.19 * *$ & $-0.67 * *$ & $-0.43 * *$ & $-0.19 * *$ \\
\hline & \multicolumn{2}{|c|}{ PL } & PT & PLE & NSF & DMC \\
\hline 001 & \multicolumn{2}{|c|}{$0.03^{\mathrm{ns}}$} & $0.03 * *$ & $0.27 * *$ & $16.50^{* *}$ & $-1.29 * *$ \\
\hline 004 & \multicolumn{2}{|c|}{$0.09 * *$} & $0.03 * *$ & $0.53 * *$ & $17.97 * *$ & $-0.66^{\mathrm{ns}}$ \\
\hline 77.3 & \multicolumn{2}{|c|}{$-0.13 * *$} & $-0.04 * *$ & $-0.64 * *$ & $-21.49 * *$ & $-0.14^{\mathrm{ns}}$ \\
\hline 099 & \multicolumn{2}{|c|}{$0.07 * *$} & $0.02 * *$ & $0.53 * *$ & $13.78 * *$ & $-1.02 * *$ \\
\hline 134 & \multicolumn{2}{|c|}{$-0.02^{\mathrm{ns}}$} & $-0.01 *$ & $-0.22 * *$ & $-1.27^{\mathrm{ns}}$ & $1.64 * *$ \\
\hline 137 & \multicolumn{2}{|c|}{$0.09 * *$} & $-0.01^{\text {ns }}$ & $0.03^{\text {ns }}$ & $-5.94 * *$ & $0.77 *$ \\
\hline 390 & \multicolumn{2}{|c|}{$-0.12 * *$} & $-0.04 * *$ & $-0.47 * *$ & $-19.55 * *$ & $0.71^{\mathrm{ns}}$ \\
\hline
\end{tabular}

${ }^{n s}$ Not significant, **and* Significant at 1 and $5 \%$ of error probability, respectively by Student's t-test. $\mathrm{DFR}=$ days to fructification; $\mathrm{NFP}=$ number of fruits per plant; $\mathrm{FW}=$ fruit weight; $\mathrm{FL}=$ fruit length; $\mathrm{LFD}=$ largest fruit diameter; $\mathrm{SFD}=$ smallest fruit diameter; $\mathrm{PL}=$ pedicel length; $\mathrm{PT}=$ pericarp thickness; $\mathrm{PLE}=$ placenta length; $\mathrm{NFS}=$ number of seeds per fruit; $\mathrm{DMC}=$ dry matter content. 
reported by Pessoa et al. (2019a) in pepper plants.

For the trait days to fructification, genotypes UFPB77.3 and UFPB390 presented superior and negative values of the general combining ability (ĝi) estimates and a differentiated behavior from the remaining genotypes evaluated (Table 1). These genotypes are recommended for selection since they showed to be precocious, reducing the time to fructification, with the possibility to obtain precocious plants in crosses in which they take part. Precocity is an important characteristic in ornamental plants since genotypes that produce fruits faster are soon commercialized, which is interesting for producers. The effects of the general combining ability inform of the potentialities of parent individuals in generating favorable combinations for the formation of predominantly additive genes (Baldissera et al., 2014).

For number of fruits per plant, fruit weight, fruit length, largest fruit diameter, and smallest fruit diameter, genotypes UFPB77.3 and UFPB390 are indicated for crosses in order to obtain promising populations for the development of fruits with ornamental aspect. Since these parents showed the major negatives ĝi values and for ornamental purposes smaller fruits are needed. Parents of interest must be used in order to increase the production per plant as well as to decrease the fruit size (Nascimento et al., 2014). Pessoa et al. (2018), in a study of genetic divergence between pepper genotypes, recommended the selection of genotypes UFPB 77.3 and UFPB390 for fruit traits since they presented the highest number of fruits per plant and small fruits. In ornamental peppers, the selection of smaller fruits is recommended in order to maintain the balance with plant architecture (Fortunato et al., 2019).

The highest positive values of the GCA (ĝi) estimates for pericarp thickness and placenta length were presented by genotypes UFPB001, UFPB004, and UFPB099 (Table 1). These traits are important in the study of pepper plants since the largest amount of capsaicin is present in the placenta, in the seeds, and in the pericarp of fruits (Rêgo et al., 2012), thus, if the purpose is to obtain ornamental pepper fruits with the highest pungency content, these genotypes are indicated for selection.

By the gi values referring to pedicel length, the accessions UFPB004 and UFPB137 presented the highest positive values of GCA (Table 1). For this trait, these genotypes are recommended for selection. For pepper plants with ornamental purposes, it is interesting to select fruits with longer pedicels so that they are prominent on the leaves and facilitate fruit harvest (Nascimento et al., 2019b).

Genotypes UFPB001, UFPB004, and UFPB099 probably gather favorable alleles for the number of seeds per fruits since they present the highest positive

Table 2. Estimates of the specific combining ability $\left(\mathrm{S}_{\mathrm{ij}}\right)$ for fruit traits in Capsicum annuum. Areia, UFPB, 2019.

\begin{tabular}{|c|c|c|c|c|c|c|c|c|}
\hline \multirow{3}{*}{$\begin{array}{l}\begin{array}{l}\text { UFPB } \\
\text { (hybrids/reciprocals) }\end{array} \\
001 \times 004(004 \text { x 001) }\end{array}$} & \multicolumn{8}{|c|}{ Characters } \\
\hline & \multicolumn{2}{|c|}{ DFR } & \multicolumn{2}{|c|}{ NFP } & \multicolumn{2}{|c|}{ FW } & \multicolumn{2}{|c|}{ FL } \\
\hline & $-0.79^{\text {ns }}$ & $2.06^{* *}$ & $12.03 * *$ & $-0.18^{\mathrm{ns}}$ & $1.40 * *$ & $0.27^{\mathrm{ns}}$ & $-0.23 * *$ & $-0.01^{\mathrm{ns}}$ \\
\hline $001 \times 099(099 \times 001)$ & $2.32 * *$ & $-1.43^{*}$ & $8.63 * *$ & $1.37^{\mathrm{ns}}$ & $0.86^{* *}$ & $-0.38^{*}$ & $-0.36^{* *}$ & $-0.25^{* *}$ \\
\hline $004 \times 099(099 \times 004)$ & $1.74^{*}$ & $-2.25 * *$ & $7.82 * *$ & $-1.18^{\mathrm{ns}}$ & $1.11^{* *}$ & $-0.17^{\mathrm{ns}}$ & $-0.23^{* *}$ & $0.04^{\mathrm{ns}}$ \\
\hline $77.3 \times 001(001 \times 77.3)$ & $0.61^{\mathrm{ns}}$ & $-5.56^{* *}$ & $-16.64 *$ & $3.75^{\text {ns }}$ & $-1.19^{* *}$ & $0.29^{\mathrm{ns}}$ & $0.11^{\mathrm{ns}}$ & $0.15^{*}$ \\
\hline 77.3 x 004(004 x 77.3) & $-3.01 * *$ & $1.31^{\mathrm{ns}}$ & $-15.89 *$ & $0.87^{\mathrm{ns}}$ & $-1.16^{* *}$ & $-0.01^{\mathrm{ns}}$ & $0.23 * *$ & $-0.04^{\mathrm{ns}}$ \\
\hline 77.3 x 099(099 x 77.3) & $-5.65 * *$ & $-3.68 * *$ & $-14.61^{*}$ & $-2.00^{\mathrm{ns}}$ & $-0.99 * *$ & $-0.26^{\mathrm{ns}}$ & $0.12^{\mathrm{ns}}$ & $-0.12^{\mathrm{ns}}$ \\
\hline $134 \times 137(137 \times 134)$ & $3.38^{* *}$ & $4.93^{* *}$ & $-5.94 *$ & $-7.00 *$ & $-0.17^{\mathrm{ns}}$ & $0.28^{\mathrm{ns}}$ & $-0.24 *$ & $0.26^{* *}$ \\
\hline $134 \times 390(390 \times 134)$ & $-0.18^{\text {ns }}$ & $4.75^{* *}$ & $-0.59^{\text {ns }}$ & $9.00 * *$ & $0.27^{\mathrm{ns}}$ & $0.12^{\mathrm{ns}}$ & $-0.21 * *$ & $0.09^{\mathrm{ns}}$ \\
\hline $134 \times 77.3(77.3 \times 134)$ & $-1.62 *$ & $2.93 * *$ & $7.72 * *$ & $-2.68^{\mathrm{ns}}$ & $0.28^{\mathrm{ns}}$ & $0.09^{\text {ns }}$ & $-0.27 * *$ & $0.10^{\mathrm{ns}}$ \\
\hline $134 \times 001(001 \times 134)$ & $-1.40 *$ & $3.56^{* *}$ & $-0.66^{\mathrm{ns}}$ & $1.68^{\mathrm{ns}}$ & $0.21^{\mathrm{ns}}$ & $0.14^{\mathrm{ns}}$ & $0.40 * *$ & $0.09^{\mathrm{ns}}$ \\
\hline $134 \times 004(004 \times 134)$ & $-2.03 * *$ & $4.68^{* *}$ & $2.83^{\mathrm{ns}}$ & $-1.31^{*}$ & $-0.57 * *$ & $0.21^{\mathrm{ns}}$ & $0.19 * *$ & $0.02^{\mathrm{ns}}$ \\
\hline $134 \times 099(099 \times 134)$ & $-1.67^{*}$ & $2.93^{* *}$ & $4.56^{*}$ & $-5.12 *$ & $0.23^{\text {ns }}$ & $0.11^{\mathrm{ns}}$ & $0.37 * *$ & $0.12^{\mathrm{ns}}$ \\
\hline $137 \times 390(390 \times 137)$ & $-0.34^{\mathrm{ns}}$ & $-2.81 * *$ & $0.25^{\mathrm{ns}}$ & $7.00 *$ & $0.41 *$ & $0.01^{\mathrm{ns}}$ & $-0.01^{\mathrm{ns}}$ & $-0.02^{\mathrm{ns}}$ \\
\hline $137 \times 77.3(77.3 \times 390)$ & $-1.59^{*}$ & $-0.81^{\text {ns }}$ & $-2.68^{\mathrm{ns}}$ & $-2.56^{\mathrm{ns}}$ & $0.79 * *$ & $-0.18^{\text {ns }}$ & $0.22 * *$ & $-0.04^{\mathrm{ns}}$ \\
\hline $137 \times 001(001 \times 137)$ & $-0.62^{\mathrm{ns}}$ & $0.06^{\mathrm{ns}}$ & $1.49^{\mathrm{ns}}$ & $3.62^{\mathrm{ns}}$ & $-0.92 * *$ & $0.05^{\mathrm{ns}}$ & $0.04^{\mathrm{ns}}$ & $0.06^{\mathrm{ns}}$ \\
\hline $137 \times 004(004 \times 137)$ & $-1.01^{\mathrm{ns}}$ & $1.68^{*}$ & $2.37^{\mathrm{ns}}$ & $0.12^{\mathrm{ns}}$ & $-0.23^{\mathrm{ns}}$ & $-0.05^{\mathrm{ns}}$ & $0.18 * *$ & $-0.01^{\mathrm{ns}}$ \\
\hline $137 \times 099(099 \times 137)$ & $0.60^{\text {ns }}$ & $1.06^{\mathrm{ns}}$ & $-2.65^{\mathrm{ns}}$ & $0.93^{\text {ns }}$ & $0.69^{* *}$ & $-0.92 * *$ & $0.52 * *$ & $-0.25^{* *}$ \\
\hline $390 \times 77.3(77.3 \times 390)$ & $-0.29^{\mathrm{ns}}$ & $-0.75^{\mathrm{ns}}$ & $23.49^{* *}$ & $-14.80^{*}$ & $1.12 * *$ & $-0.13^{\mathrm{ns}}$ & $-0.09^{\text {ns }}$ & $-0.19^{*}$ \\
\hline $390 \times 001(001 \times 390)$ & $-1.76^{*}$ & $-2.93 * *$ & $-16.20^{*}$ & $-1.81^{\mathrm{ns}}$ & $-1.08^{* *}$ & $0.04^{\mathrm{ns}}$ & $0.30^{* *}$ & $0.02^{\mathrm{ns}}$ \\
\hline $390 \times 004(004 \times 390)$ & $-3.01 * *$ & $1.06^{\mathrm{ns}}$ & $-17.14 *$ & $0.12^{\text {ns }}$ & $-0.52 * *$ & $-0.94^{\mathrm{ns}}$ & $0.29 * *$ & $-0.15^{*}$ \\
\hline $390 \times 099(099 \times 390)$ & $-1.84 * *$ & $-0.12^{\mathrm{ns}}$ & $-8.42 * *$ & $3.68^{\text {ns }}$ & $-1.06^{* *}$ & $-0.17^{\mathrm{ns}}$ & $0.14^{*}$ & $-0.18^{*}$ \\
\hline
\end{tabular}


Tabela 2. Continuation

\begin{tabular}{|c|c|c|c|c|c|c|c|c|}
\hline \multirow{3}{*}{$\begin{array}{l}\begin{array}{l}\text { UFPB } \\
\text { (hybrids/reciprocals) }\end{array} \\
001 \times 004(004 \text { x 001) }\end{array}$} & \multicolumn{8}{|c|}{ Characters } \\
\hline & \multicolumn{2}{|c|}{ LFD } & \multicolumn{2}{|c|}{ SFD } & \multicolumn{2}{|c|}{ PL } & \multicolumn{2}{|c|}{ PT } \\
\hline & $0.14 * *$ & $0.07 *$ & $0.12 * *$ & $0.04^{\mathrm{ns}}$ & $-0.05^{\mathrm{ns}}$ & $-0.02^{\mathrm{ns}}$ & $0.04^{\mathrm{ns}}$ & $0.01^{\mathrm{ns}}$ \\
\hline $001 \times 099(099 \times 001)$ & $0.14 * *$ & $0.06^{\mathrm{ns}}$ & $0.09 * *$ & $0.00^{\mathrm{ns}}$ & $-0.10 * *$ & $-0.07^{\mathrm{ns}}$ & $0.03 * *$ & $-0.02 * *$ \\
\hline 004 x $099(099 \times 004)$ & $0.06^{*}$ & $-0.06^{\mathrm{ns}}$ & $0.08 * *$ & $-0.01^{\mathrm{ns}}$ & $0.04^{\mathrm{ns}}$ & $0.08 *$ & $-0.02^{\mathrm{ns}}$ & $-0.00^{\mathrm{ns}}$ \\
\hline $77.3 \times 001(001 \times 77.3)$ & $-0.15 * *$ & $0.06^{\mathrm{ns}}$ & $-0.08 * *$ & $0.04^{\mathrm{ns}}$ & $0.12 * *$ & $0.10^{*}$ & $-0.01 *$ & $0.00^{\mathrm{ns}}$ \\
\hline $77.3 \times 004(004 \times 77.3)$ & $-0.05^{\mathrm{ns}}$ & $0.01^{\mathrm{ns}}$ & $-0.12 * *$ & $-0.01^{\mathrm{ns}}$ & $0.11 * *$ & $-0.02^{\mathrm{ns}}$ & $0.01^{\mathrm{ns}}$ & $-0.01^{\mathrm{ns}}$ \\
\hline $77.3 \times 099(099 \times 77.3)$ & $-0.10 * *$ & $-0.04^{\mathrm{ns}}$ & $-0.08 * *$ & $0.01^{\mathrm{ns}}$ & $0.02^{\mathrm{ns}}$ & $-0.12 * *$ & $-0.01 *$ & $0.01^{\mathrm{ns}}$ \\
\hline 134 x $137(137$ x 134) & $-0.03^{\mathrm{ns}}$ & $0.04^{\mathrm{ns}}$ & $-0.02^{\mathrm{ns}}$ & $0.01^{\mathrm{ns}}$ & $0.01^{\mathrm{ns}}$ & $-0.04^{\mathrm{ns}}$ & $-0.01^{\mathrm{ns}}$ & $0.01^{\mathrm{ns}}$ \\
\hline 134 x $390(390 \times 134)$ & $0.03^{\mathrm{ns}}$ & $0.09 *$ & $0.05^{*}$ & $0.00^{\mathrm{ns}}$ & $-0.06^{\mathrm{ns}}$ & $0.17 * *$ & $0.04^{\mathrm{ns}}$ & $0.01^{\mathrm{ns}}$ \\
\hline $134 \times 77.3(77.3 \times 134)$ & $0.01 * *$ & $0.03^{\mathrm{ns}}$ & $0.09 * *$ & $0.02^{\mathrm{ns}}$ & $-0.02^{\mathrm{ns}}$ & $0.07^{\mathrm{ns}}$ & $0.01^{\mathrm{ns}}$ & $0.01^{\mathrm{ns}}$ \\
\hline 134 x $001(001$ x 134) & $0.05^{\mathrm{ns}}$ & $-0.02^{\mathrm{ns}}$ & $-0.02^{\mathrm{ns}}$ & $-0.01^{\mathrm{ns}}$ & $0.07 *$ & $0.03^{\mathrm{ns}}$ & $0.01^{\mathrm{ns}}$ & $0.01^{\mathrm{ns}}$ \\
\hline 134 x $004(004 \times 134)$ & $-0.05^{\mathrm{ns}}$ & $0.06^{\mathrm{ns}}$ & $-0.09 * *$ & $0.02^{\mathrm{ns}}$ & $0.09 *$ & $0.13 * *$ & $0.00^{\mathrm{ns}}$ & $0.01^{\mathrm{ns}}$ \\
\hline $134 \times 099(099 \times 134)$ & $0.02^{\mathrm{ns}}$ & $0.01^{\mathrm{ns}}$ & $-0.01^{\mathrm{ns}}$ & $0.02^{\mathrm{ns}}$ & $0.05^{\mathrm{ns}}$ & $0.06^{\mathrm{ns}}$ & $-0.01 *$ & $-0.01^{\mathrm{ns}}$ \\
\hline 137 x $390(390$ x 137) & $0.08 * *$ & $-0.01^{\mathrm{ns}}$ & $0.05 *$ & $-0.01^{\mathrm{ns}}$ & $-0.06^{\mathrm{ns}}$ & $0.13 * *$ & $0.01^{\mathrm{ns}}$ & $-0.03^{\mathrm{ns}}$ \\
\hline $137 \times 77.3(77.3 \times 390)$ & $0.07 *$ & $-0.01^{\mathrm{ns}}$ & $0.01^{\mathrm{ns}}$ & $0.01^{\mathrm{ns}}$ & $-0.14 * *$ & $0.02^{\mathrm{ns}}$ & $0.01^{\mathrm{ns}}$ & $-0.02^{\mathrm{ns}}$ \\
\hline 137 x 001(001 x 137) & $-0.14 * *$ & $0.01^{\mathrm{ns}}$ & $-0.05^{*}$ & $-0.02^{\mathrm{ns}}$ & $0.05^{\mathrm{ns}}$ & $0.18 * *$ & $-0.01 *$ & $0.08^{\mathrm{ns}}$ \\
\hline 137 x $004(004$ x 137) & $-0.03^{\mathrm{ns}}$ & $0.01^{\mathrm{ns}}$ & $-0.03^{\mathrm{ns}}$ & $-0.03^{\mathrm{ns}}$ & $0.15^{* *}$ & $0.03^{\mathrm{ns}}$ & $0.01^{\mathrm{ns}}$ & $0.01^{\mathrm{ns}}$ \\
\hline 137 x $099(099 \times 137)$ & $0.09 * *$ & $-0.10^{*}$ & $0.03^{\mathrm{ns}}$ & $0.02^{\mathrm{ns}}$ & $0.12 * *$ & $-0.03^{\mathrm{ns}}$ & $0.01^{\mathrm{ns}}$ & $-0.01^{\mathrm{ns}}$ \\
\hline $390 \times 77.3(77.3 \times 390)$ & $0.12 * *$ & $-0.05^{\mathrm{ns}}$ & $0.05 *$ & $0.01^{\mathrm{ns}}$ & $-0.02^{\mathrm{ns}}$ & $-0.13 * *$ & $0.01 *$ & $-0.01 *$ \\
\hline 390 x $001(001$ x 390) & $-0.19 * *$ & $-0.01^{\mathrm{ns}}$ & $-0.11 * *$ & $-0.03^{\mathrm{ns}}$ & $-0.02^{\mathrm{ns}}$ & $0.02^{\mathrm{ns}}$ & $-0.02 * *$ & $0.00^{\mathrm{ns}}$ \\
\hline $390 \times 004(004 \times 390)$ & $-0.02^{\mathrm{ns}}$ & $-0.12 *$ & $-0.05^{*}$ & $-0.06^{*}$ & $0.09 *$ & $0.01^{\mathrm{ns}}$ & $0.00^{\mathrm{ns}}$ & $-0.01^{\mathrm{ns}}$ \\
\hline 390 x $099(099 \times 390)$ & $-0.17^{*}$ & $-0.05^{\mathrm{ns}}$ & $-0.12 * *$ & $-0.02^{\mathrm{ns}}$ & $0.03^{\mathrm{ns}}$ & $0.05^{\mathrm{ns}}$ & $-0.01 * *$ & $0.01^{\mathrm{ns}}$ \\
\hline
\end{tabular}

Tabela 2. Continuation

\begin{tabular}{|c|c|c|c|c|c|c|}
\hline \multirow{3}{*}{$\begin{array}{l}\text { UFPB (hybrids/reciprocals) } \\
001 \text { x } 004 \text { (004 x 001) }\end{array}$} & \multicolumn{6}{|c|}{ Characters } \\
\hline & \multicolumn{2}{|c|}{ PLE } & \multicolumn{2}{|c|}{ NSF } & \multicolumn{2}{|c|}{ DMC } \\
\hline & $-0.30 * *$ & $-0.19^{*}$ & $6.94 * *$ & $5.41 *$ & $-0.11^{\mathrm{ns}}$ & $-0.85^{\mathrm{ns}}$ \\
\hline $001 \times 099(099 \times 001)$ & $-0.35 * *$ & $-0.19 *$ & $4.25^{\mathrm{ns}}$ & $-2.66^{\mathrm{ns}}$ & $-1.62^{\mathrm{ns}}$ & $0.35^{\mathrm{ns}}$ \\
\hline 004 x $099(099 \times 004)$ & $-0.17^{*}$ & $-0.04^{\mathrm{ns}}$ & $5.06^{\mathrm{ns}}$ & $-6.97 *$ & $-1.46^{\mathrm{ns}}$ & $-1.25^{\mathrm{ns}}$ \\
\hline $77.3 \times 001(001 \times 77.3)$ & $0.02^{\mathrm{ns}}$ & $0.17 *$ & $-5.29 *$ & $11.33 * *$ & $0.43^{\mathrm{ns}}$ & $0.58^{\mathrm{ns}}$ \\
\hline $77.3 \times 004(004 \times 77.3)$ & $0.32 * *$ & $-0.13^{\mathrm{ns}}$ & $9.19 * *$ & $2.08^{\mathrm{ns}}$ & $0.31^{\mathrm{ns}}$ & $-1.42^{\mathrm{ns}}$ \\
\hline $77.3 \times 099(099 \times 77.3)$ & $0.25 * *$ & $-0.16^{*}$ & $2.38^{\mathrm{ns}}$ & $-12.21 * *$ & $1.91^{*}$ & $1.64^{\mathrm{ns}}$ \\
\hline $134 \times 137(137 \times 134)$ & $-0.22 * *$ & $0.08^{\mathrm{ns}}$ & $-1.02^{\mathrm{ns}}$ & $-1.81^{\mathrm{ns}}$ & $0.15^{\mathrm{ns}}$ & $-0.14^{\mathrm{ns}}$ \\
\hline $134 \times 390(390 \times 134)$ & $-0.10^{\mathrm{ns}}$ & $0.15^{*}$ & $-3.50^{\mathrm{ns}}$ & $6.14^{*}$ & $0.48^{\mathrm{ns}}$ & $0.25^{\mathrm{ns}}$ \\
\hline $134 \times 77.3(77.3 \times 134)$ & $-0.25^{*}$ & $0.07^{\mathrm{ns}}$ & $-2.17^{\mathrm{ns}}$ & $4.64^{\mathrm{ns}}$ & $-0.30^{\mathrm{ns}}$ & $-1.92 *$ \\
\hline $134 \times 001(001 \times 134)$ & $0.29 * *$ & $0.07^{\mathrm{ns}}$ & $3.67^{\mathrm{ns}}$ & $2.79^{\mathrm{ns}}$ & $0.44^{\mathrm{ns}}$ & $0.77^{\mathrm{ns}}$ \\
\hline $134 \times 004(004 \times 134)$ & $0.23 * *$ & $0.01^{\mathrm{ns}}$ & $4.41^{\mathrm{ns}}$ & $13.37 *$ & $1.54^{\mathrm{ns}}$ & $1.29^{\mathrm{ns}}$ \\
\hline $134 \times 099(099 \times 134)$ & $0.28 * *$ & $0.24 * *$ & $11.69 * *$ & $2.20^{\mathrm{ns}}$ & $-1.74^{*}$ & $0.57^{\mathrm{ns}}$ \\
\hline $137 \times 390(390 \times 137)$ & $-0.05^{\mathrm{ns}}$ & $0.11 *$ & $4.73 *$ & $3.04^{\mathrm{ns}}$ & $0.15^{\mathrm{ns}}$ & $-1.96^{*}$ \\
\hline $137 \times 77.3(77.3 \times 390)$ & $0.13^{\mathrm{ns}}$ & $-0.01^{\mathrm{ns}}$ & $0.91^{\mathrm{ns}}$ & $2.52^{\mathrm{ns}}$ & $0.30^{\mathrm{ns}}$ & $-0.92^{\mathrm{ns}}$ \\
\hline $137 \times 001(001 \times 137)$ & $0.31 * *$ & $-0.07^{\mathrm{ns}}$ & $-9.79 * *$ & $18.14 * *$ & $1.53^{\mathrm{ns}}$ & $-0.41^{\mathrm{ns}}$ \\
\hline $137 \times 004(004 \times 137)$ & $0.13^{\mathrm{ns}}$ & $-0.17^{*}$ & $4.32^{\mathrm{ns}}$ & $-0.22^{\mathrm{ns}}$ & $-0.19^{\mathrm{ns}}$ & $0.57^{\mathrm{ns}}$ \\
\hline $137 \times 099(099 \times 137)$ & $0.34 * *$ & $-0.18 *$ & $2.45^{\mathrm{ns}}$ & $-5.91 *$ & $0.02^{\mathrm{ns}}$ & $-0.69^{\mathrm{ns}}$ \\
\hline $390 \times 77.3(77.3 \times 390)$ & $-0.13^{\mathrm{ns}}$ & $-0.19 *$ & $-2.47^{\mathrm{ns}}$ & $-5.22^{\mathrm{ns}}$ & $-0.24^{\mathrm{ns}}$ & $0.03^{\mathrm{ns}}$ \\
\hline
\end{tabular}


Tabela 2. Continuation

\begin{tabular}{|c|c|c|c|c|c|c|}
\hline \multirow{2}{*}{ UFPB (hybrids/reciprocals) } & \multicolumn{6}{|c|}{ Characters } \\
\hline & \multicolumn{2}{|c|}{ PLE } & \multicolumn{2}{|c|}{ NSF } & \multicolumn{2}{|c|}{ DMC } \\
\hline $390 \times 001(001 \times 390)$ & $0.17 *$ & $0.09^{\text {ns }}$ & $-0.32^{\mathrm{ns}}$ & $-1.69^{\mathrm{ns}}$ & $-1.63 *$ & $0.86^{\mathrm{ns}}$ \\
\hline $390 \times 004(004 \times 390)$ & $0.28 * *$ & $-0.19 *$ & $4.84^{*}$ & $-7.60 *$ & $1.19^{\mathrm{ns}}$ & $0.38^{\text {ns }}$ \\
\hline $390 \times 099(099 \times 390)$ & $0.12^{\text {ns }}$ & $-0.30 * *$ & $-1.04^{\mathrm{ns}}$ & $-6.77 *$ & $2.31 * *$ & $-0.25^{\mathrm{ns}}$ \\
\hline
\end{tabular}

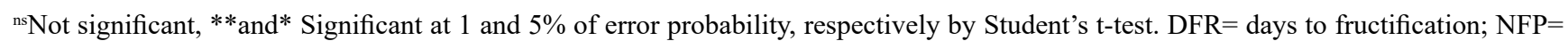
number of fruits per plant; $\mathrm{FW}=$ fruit weight; $\mathrm{FL}=$ fruit length; $\mathrm{LFD}=$ largest fruit diameter; $\mathrm{SFD}=$ smallest fruit diameter; $\mathrm{PL}=$ pedicel length; $\mathrm{PT}=$ pericarp thickness; $\mathrm{PLE}=$ placenta length; $\mathrm{NSF}=$ number of seeds per fruit; $\mathrm{DMC}=$ dry matter content.

values (Table 1). This trait is related to the production component, and it is interesting that pepper parents present higher values of the gi estimate for the number of seeds per fruit (Nascimento et al., 2014) and also better performances for the remaining fruit-related traits.

For the dry matter content in pepper fruits, genotype UFPB134 presented the highest positive value of the estimate of the general combining ability (Table 1), a desirable trait since the fruits of these species are used either in natura or dehydrated. This result is important since it indicates that this parent presents favorable effects for this trait and can be employed in crosses, for that purpose.

Considering the effects of the specific combining ability $\left(\mathrm{S}_{\mathrm{ij}}\right)$ referring to the days to fructification, the hybrid combinations that presented the highest negative estimates for this trait were UFPB77.3 x UFPB099, UFPB001 $x$ UFPB77.3, UFPB390 x UFPB004, and UFPB099 x UFPB77.3, with major values of $-5.65,-5.56,-3.01$ and -3.68 , respectively (Table 2 ). Thus, indicating the possibility to obtain lineages more precocious. It is worth noting that parents UFPB77.3 and UFPB390 showed to be the most precocious according to the $g i$ estimates. Griffing (1956) reported that the best hybrid combinations present high SCA effects, either positive or negative, and that crosses should occur between divergent parents, in which at least one of them should present a high GCA value, as observed in this research.

The hybrid UFPB390 x UFPB77.3 presented the highest positive and significant SCA value $\left(\mathrm{S}_{\mathrm{ij}}\right)$ for the number of fruits per plant (23.49) (Table 2 ). The higher the number of fruits per plant, the more attractive is the plant to the customer, considering that fruits are essential in pepper plants with ornamental purposes. Similar results were obtained by Hasanuzzaman et al. (2013) in C. annuum. Neitzke et al. (2016) reported that the most promising pepper plants to become successful ornamental cultivars are those that present a higher number of fruits contrasting with the foliage.

For the fruit weight, the hybrid combinations UFPB77.3 x UFPB001, UFPB77.3 x UFPB004, UFPB390 x UFPB001, and UFPB390 x UFPB099 presented the best values of significant and negative estimates of $\mathrm{S}_{\mathrm{ij}}$ of -1.19 , $-1.16,-1.08$ and -1.06 , respectively, indicating the opportunity to diminish the fruit weight which is interesting in ornamental pepper (Table 2). Fruit weight is a trait correlated with fruit length (Moulin et al., 2015) and lighter and smaller fruits are ideal for ornamentation due to the small size of pepper plants (Silva et al., 2015).

Regarding the SCA estimates (Sij) for fruit length, hybrids UFPB134 $x$ UFPB77.3 (-0.27), UFPB099 $x$ UFPB 137 (-0.25), UFPB134 x UFPB137 (-0.24), and UFPB134 x UFPB390 (-0.21) (Table 2) were significant. Pepper fruits recommended for ornamental purposes are usually small, elongated, and erect (Melo et al., 2014), then, the hybrids with major negative values for this trait should be selected with the goal of diminishing fruit length.

The $S_{i j}$ results referring to the largest and smallest fruit diameter showed that the UFPB390 $\times$ UFPB001 and UFPB390 x UFPB099 combinations presented the highest negative significant estimates for these traits, of -0.19 and -0.17 , respectively (Table 2 ). Fruit diameter and length are important in the harmonization of ornamental pepper plants (Silva et al., 2015). Fruit selection based on these traits was approached in other studies with ornamental peppers. Authors demonstrated the importance of selecting plants with small fruits (Pessoa et al., 2018).

The highest significant positive $S_{\text {. }}$ values were detected in the UFPB 137 $\mathrm{x}$ UFPB004 (0.15) and UFPB001 $\mathrm{x}$ UFPB137 (0.18) crosses, for pedicel length (Table 2). This is an interesting trait for ornamentation since fruits with longer pedicel length are indicated for cultivation for both pot ornamental purposes and floral arrangements (Melo et al., 2014).

The hybrid UFPB001 x UFPB099 $(0.03)$ presented the highest significant positive value of the specific combining ability for pericarp thickness (Table 2). This trait influences the increase of fruit firmness (Pessoa et al., 2018), and the fruits remain longer on the plant. This trait adds economic values to the species if it is also used for in natura consumption, as well as in the form of condiments, sauces, paprika, and others (Abud et al., 2018).

Regarding the placenta length trait, the UFPB137 x UFPB099 (0.34) and UFPB77.3 x UFPB004 (0.32) combinations presented the highest SCA estimates $\left(\mathrm{S}_{\mathrm{iij}}\right)$ (Table 2). Hybrid combinations UFPB134 x UFPB099 (11.69), UFPB004 x UFPB134 (13.37), UFPB001 x UFPB137 (18.14), and UFPB001 x UFPB77.3 (11.33) presented the highest positive estimates of the SCA for number of seeds per fruits (Table 2). The hybridization between varieties of the same species usually produces numerous seeds (Rêgo et al., 2011).

The highest significant positive and negative value for the fruit dry 
matter content was observed in hybrids UFPB390 x UFPB099 (2.31) and UFPB77.3 x UFPB099 (1.91), respectively (Table 2). Rego et al. (2011) reported that this trait is directly correlated with the size and weight of fruits, and thus recommend the selection of hybrids that present the highest negative values.

UFPB77.3 and UFPB390 should be chosen as parents since they presented the major negative gi values for traits such as fruit length, major and minor fruit width, and fruit weight. These two genitors also showed major positive values for fruit number per plant and they are the parents of the best hybrid combinations.

Based on the exposed in the early paragraph and also in the specific combining ability values, showed in the results section, the hybrids UFPB77.3 x UFPB099, UFPB390 x UFPB77.3, UFPB390 x UFPB001, UFPB390 x UFPB099, UFPB134 x UFPB77.3 and UFPB134 x UFPB390 should be selected.

For those traits that showed predominance of additive effects (fruit length, smallest fruit diameter, pedicel and placenta length) the use of pedigree improvement method must be lead to select elite lineages and release new varieties. For those traits where nonadditive effects were predominant (days to fructification, number of fruits per plant, fruit weight, largest fruit diameter, pericarp thickness and dry matter content) there is an opportunity for developing F1 hybrids.

\section{REFERENCES}

ABUD, HF; ARAUJO, RF; PINTO, CMF; ARAUJO, EF; ARAUJO, AV; SANTOS, JA. 2018. Caraterização morfométrica dos frutos de pimentas malagueta e biquinho. Revista Brasileira de Agropecuária Sustentável 8: 29-39.

BALDISSERA, JNC; VALENTINI, G; CIAN, MM; ALMEIDA, CB; GUIDOLIN, AF; COIMBRA, JLM. 2012. Combining ability and reciprocal effect on agronomical traits of bean. Semina: Ciências Agrárias 33: 471-480.

BALDISSERA, JNC; VALENTINI, G; COAN, MMD; GUIDOLIN, AF; COIMBRA, JLM. 2014. Fatores genéticos relacionados com a herança em populações de plantas autógamas.
Revista de Ciências Agroveterinárias 13: 181-189.

BOSLAND, PW; VOTAVA, EJ. 2012. Peppers: vegetable and spice capsicums. CABI, Oxon, UK, 248p.

CAMARA. SJ. 2020. Engineering cayenne pepper extract as alternative biostain in microscopy. International Journal of Scientific \& Technology Research 9: 3210-3212.

CRUZ, CD. 2006. Programa genes (versão Windows): aplicativo computacional em genética e estatística. Viçosa: Editora UFV. 382p.

FORTUNATO, FLG; RÊGO, ER; CARVALHO, MG; SANTOS, CAP; RÊGO, MM. 2019. Genetic diversity in ornamental pepper plants. Comunicata Scientiae 10: 364-375.

GRIFFING, B. 1956. Concept of general and specific combining ability in relation to diallel crossing systems. Australian Journal of Biological Sciences 9: 463-493.

HASANUZZAMAN, M; HAKIM, MA; HANAFI, MM; SHUKOR-JURAIMI, A; ISLAM, MM; SHAMSUDDIN, AKM. 2013. Study of heterosis in Bangladeshi chili (Capsicum annuum L.) landraces. Agrociência 47: 683-690.

HEISER, CBJR; PICKERSGILL, B. 1969. Names for the cultivated Capsicum species (Solanaceae), Taxon 18: 277-283.

IPGRI (International Plant Genetic Resources Institute). 1995. Descriptores para Capsicum (Capsicum spp.). Roma: IPGRI: 1-51.

MELO, LF; GOMES, RLF; SILVA, VB; MONTEIRO, ER; LOPES. ACA; PERON, AP. 2014. Potencial ornamental de acessos de pimenta. Ciência Rural 44: 2010-2015.

MOULIN, MM; RODRIGUES, R; BENTO, CS; GONÇALVES, LSA; SANTOS, JO; SUNDRÉ, CP; VIANA, AP. 2015. Genetic dissection of agronomic traits in Capsicum baccatum var. pendulum. Genetics and Molecular Research 14: 2122-2132.

NASCIMENTO, NFF; RÊG， ER; NASCIMENTO, MF; BRUCKNER, CH; FINGER, FL; RÊGO, MM. 2014. Combining ability for yield and fruit quality in the pepper Capsicum annuum. Genetics and Molecular Research 13: 3237-3249.

NASCIMENTO, NFF; REGO, ER; NASCIMENTO, MF; BRUCKNER, CH; FINGER, FL; RÊGO, MM. 2019a. Evaluation of production and quality traits in interspecific hybrids of ornamental pepper. Horticultura Brasileira 37: 315-323.

NA S CIMENTO, MF ; RÊGO, ER; NASCIMENTO, NFF; LEITE, PSS; FINGER, FL; BRUCKNER, CH; RÊGO, MM. 2019 b. Heritability of morpho-agronomic traits in ornamental pepper. Crop Breeding and Applied Biotechnology 19: 253-261.

NEITZKE, RS; FISCHER, SZ; VASCONCELOS, CS; BARBIERI, RL; TREPTOW, RO. 2016. Pimentas ornamentais: aceitação e preferências do público consumidor. Horticultura Brasileira 34: 102-109.

NEVES, CG; FREITAS, JPX; CRUZ NETO, AJ; SANTOS, LR; JESUS, ON; LEDO,
CAS; OLIVEIRA, EJ. 2014. Capacidade de combinação e heterose em maracujazeiro amarelo. Bioscience Journal 30: 757-767.

PERUCKA, I; MATERSKA, M. 2001. Phenylalanine ammonia-lyase and antioxidant activities of lipophilic fraction of fresh pepper fruits Capsicum annuum L. Innovative Food Science \& Emerging Technologies 2: 189-192.

PESSOA, AMS; RÊGO, ER; CARVALHO, MG; SANTOS, CAP; RÊGO, MM. 2018. Genetic diversity among accessions of Capsicum annuum L. through morphoagronomic characters. Genetics and Molecular Biology 17: 1-15.

PESSOA, AMS; REGO, ER; REGO, MM. 2019b. General and specific combining abilities for flower characters of Capsicum annuиm L. Journal of Experimental Agriculture International 31: 1-8.

PESSOA, AMS; RÊGO, ER; SANTOS, CAP; CARVALHO, MG; MESQUITA, JCP; RÊGO, MM. 2019a. Potential of pepper plant accessions for ornamental purposes using diallel analysis. Anais da Academia Brasileira de Ciências 91: 1-16.

PICKERSGILL, B. 1997. Genetic resources and breeding of Capsicum spp. Euphytica 96: $129-133$.

PIMENTEL, AJB; SOUZA, MA; CARNEIRO, PCS; ROCHA, JRAS; MACHADO, JC; RIBEIRO, G. 2013. Análise dialélica parcial em gerações avançadas para seleção de populações segregantes de trigo. Pesquisa Agropecuária Brasileira 48: 1555-1561.

RAMALHO, MAP; SANTOS, JB; PINTO, CABP. 2008. Genética na Agropecuária. 4. ed. Lavras: UFLA, $460 \mathrm{p}$.

RÊGO, ER; FINGER, FL; RÊGO, MM. 2012. Consumption of pepper in Brazil and its implications on nutrition and health of humans and animals. In: SALAZAR, MA; ORTEGA, JM (eds). Pepper: Nutrition, Consumption and Health. New York: Sci. Publishers. p.159-170.

RÊG， ER; NASCIMENTO, NFF; NASCIMENTO, MF; SANTOS, RM; LEITE, PSS; FINGER, FL. 2011. Caracterização fenotípica para caracteres de porte em família $\mathrm{F}_{2}$ de pimenteiras ornamentais. Horticultura Brasileira 29: 1-9.

RÊGO, ER; RÊGO, MM. 2016. Genetics and breeding of chili pepper Capsicum spp. In: RÊGO, ER; RÊGO, MM; FINGER, FL (eds). Production and breeding of chilli peppers (Capsicum spp.). Springer 1: 1-134.

RÊGO, ER; RÊGO, MM. 2018. “Ornamental Pepper", in Ornamental Crops. Switzerland: Ed. Van Huylenbroeck J. (Springer International Publishing Switzerland) 11: 529-565.

RÊGO, ER; RÊGO, MM; FINGER, FL; CRUZ, CD; CASALI, VWD. 2009. A diallel study of yield components and fruit quality in chilli pepper (Capsicum baccatum). Euphytica 168: 275-287.

ROMERO, V; LARA, JR; OTERO-ESPINAR, F; SALGADO, MH; MODOLO, NSP; BARROS, GAM. 2019. Capsaicin topical cream (8\%) for 
the treatment of myofascial pain syndrome. Revista Brasileira de Anestesiologia 69: 432-438.

SILVA, CQ; JASMIM, JM; SANTOS, JO; BENTO, CS; SUDRÉ, CP; RODRIGUES, R. 2015. Phenotyping and selecting parents for ornamental purposes in pepper accessions. Horticultura Brasileira 33: 066-073.

SOUZA, FF; DIAS, RCS; QUEIRÓZ, MA. 2013.
Capacidade de combinação de linhagens avançadas e cultivares comerciais de melancia. Horticultura Brasileira 31. 595-601.

SRINIVASANA, K. 2015. Biological activities of red pepper (Capsicum annuum) and its pungent principle capsaicin: A Review. Food Science and Nutrition 56: 1-53.

VALADARES, RN; NÓBREGA, DA; LIMA, LB; SILVA, JAS; SANTOS, AMM; MELO, RA;
MENEZES, D. 2019. Combining capacity and heterosis in eggplant hybrids under high temperatures. Horticultura Brasileira 37: 348-353.

YAMAMOTO, S; NAWATA, E. 2005. Capsicum frutescens $\mathrm{L}$. in southeast and east Asia, and its dispersal routes into Japan. Economic Botany 59:18-28. 